\title{
CAPACIDAD DEL Rhizobium DE PROMOVER EL CRECIMIENTO EN PLANTAS DE TOMATE (Lycopersicon esculentum Miller)
}

\section{PGPR CAPACITY OF Rhizobium ON Lycopersicon esculentum Miller. (TOMATO)}

\author{
Nery Santillana ${ }^{1}$, Consuelo Arellano ${ }^{2}$ y Doris Zúñiga ${ }^{3}$
}

\begin{abstract}
Resumen
El presente estudio se realizó con el objetivo de evaluar el efecto de 19 cepas de Rhizobium en la germinación y en el crecimiento de plantas de Lycopersicon esculentum. El efecto de los rizobios sobre la germinación de las semillas se determinó inoculando las semillas con suspensiones densas de rizobio y dejándolas germinar en arena esterilizada. El efecto sobre el crecimiento del cultivo se estudió en condiciones de cámara de crecimiento, utilizando suelo franco arenoso, con pH 7.3. El diseño utilizado fue completamente al azar con 21 tratamientos (19 cepas, un control sin inocular y un control con fertilización química) y cuatro repeticiones. Las cepas PEVF02, PEVF03, PEVF05, PEVF08, PEVF09, PEVF10, PEPSM12, PEPSM15 y PEPSM17 estimularon la germinación de las semillas de tomate, mientras que las cepas PEVF01, PEVF02, PEVF03, PEVF04, PEVF05, PEVF08 y PEPSM14 promovieron el crecimiento de plantas de tomate (Lycopersicon esculentum). Las cepas PEVF02 y PEVF08 tuvieron un efecto significativo positivo en la germinación y en el crecimiento de las plantas de tomate, por lo cual podrían recomendarse como potenciales PGPR en este cultivo, como alternativa para reducir el uso de fertilizantes químicos.
\end{abstract}

Palabras claves: Rhizobium, rizobacterias promotoras del crecimiento de plantas (PGPR), Lycopersicon esculentum.

\begin{abstract}
Abstrac
The aim of the present study was to evaluate the effect of 19 Rhizobium strains on seed germination and growth of Lycopersicon esculentum plants. The effect of Rhizobium on germination was determined by inoculating the seeds with dense suspensions of Rhizobium and germinating them in sterilized sand. The effect of Rhizobium on tomato plants was studied in growth chamber conditions using a sandy loam soil, with $\mathrm{pH}$ 7.3. A completely random design was used with 21 treatments (19 strains, a control without inoculating nor chemical fertilization and a control with only chemical fertilization) and four repetitions. Strains PEVF02, PEVF03, PEVF05, PEVF08, PEVF09, PEVF10, PEPSM12, PEPSM15 and PEPSM17 stimulated the germination of tomato seeds, whereas strains PEVF01, PEVF02, PEVF03, PEVF04, PEVF08 and PEPSM14 promoted the growth of tomato plants (Lycopersicon esculentum). Strains PEVF02 and PEVF08 had a significant positive effect on germination and growth of tomato plants; thus these strains could be recommended as potential PGPR for this crop, as an alternative for reducing the use of chemical fertilizers.
\end{abstract}

Key words: Rhizobium, plant growth promoting rhizobacterias (PGPR), Lycopersicon esculentum.

\section{Introducción}

Es bien conocido que un considerable número de especies bacterianas asociadas con la rizósfera de las plantas son capaces de ejercer un efecto benéfico en el crecimiento de plantas. Este grupo de bacterias llamadas rizobacterias promotoras del crecimiento en plantas (PGPR) incluye el género Rhizobium (Sessitsch et al., 2002). Estas bacterias se caracterizan por su habilidad de facilitar directa o indirectamente el desarrollo de la raíz y del follaje de las plantas. La estimulación indirecta del crecimiento de plantas incluye una variedad de mecanismos por los cuales la bacteria inhibe la acción fúngica sobre el crecimiento y desarrollo de la planta (Hassan et al., 1997; Essalmani \& Lahlou, 2003). La estimulación directa puede incluir la fijación de nitrógeno (Sessitsch et al., 2002), la producción de hormonas (Perrine et al., 2004), de enzimas (Mayak et al., 2004) de sideróforos (van Rossum et al., 1994; Carson et al., 2000) y solubilización de fosfatos (Rodriguez \& Fraga, 1999). La capacidad PGPR de Rhizobium ha sido estudiada por varias décadas (Chakravarty \& Purkayastha, 1984; Chabot et al., 1996; Hassan et al., 1997; Rodriguez \& Fraga, 1999), sin embargo, en los últimos años este estudio ha sido intensificado (Yanni et al., 2001; Essalmani \& Lahlou, 2003; Dey et al., 2004; Mhadhbi et al., 2004; Perrine et al., 2004) porque la agricultura sustentable demanda mejorar la eficiencia de la fijación de nitrógeno a través del uso de bacterias competitivas capaces de extender la ventaja de la 
simbiosis a otros cultivos no leguminosas. En tal sentido se realizó el presente estudio con el objetivo de evaluar el efecto de 19 cepas de Rhizobium en la germinación $\mathrm{y}$ en el crecimiento de plantas de Lycopersicon esculentum en condiciones de invernadero.

\section{Materiales y métodos \\ Microorganismos}

Se utilizaron 19 cepas de Rhizobium, aisladas previamente de diferentes lugares y plantas hospederas tales como haba (Vicia faba) y holantao (Pisum sativum macrocarpum) (Tabla 1).

Tabla 1. Origen, planta hospedera y colección de procedencia de cepas de Rhizobium.

\begin{tabular}{|c|c|c|c|c|}
\hline $\begin{array}{l}\text { Código } \\
\text { Cepas }\end{array}$ & Origen & $\begin{array}{l}\text { Altitud } \\
(\mathrm{msnm})\end{array}$ & $\begin{array}{c}\text { Planta } \\
\text { Hospedera }\end{array}$ & Colección \\
\hline PEVF01 & Huancavelica & 3.500 & Vicia faba & $\begin{array}{c}\text { UNSCH- } \\
\text { Ayacucho(1) }\end{array}$ \\
\hline PEVF02 & Huancavelica & 3.500 & Vicia faba & UNSCH-Ayacucho \\
\hline PEVF03 & Huancavelica & 3.500 & Vicia faba & UNSCH-Ayacucho \\
\hline PEVF04 & Huancavelica & 3.500 & Vicia faba & UNSCH-Ayacucho \\
\hline PEVF05 & Huancavelica & 3.500 & Vicia faba & UNSCH-Ayacucho \\
\hline PEVF06 & Huancavelica & 3.500 & Vicia faba & UNSCH-Ayacucho \\
\hline PEVF07 & Argentina & 200 & Pisum sativum & Argentina \\
\hline PEVF08 & Ayacucho & 2.750 & Vicia faba & UNSCH-Ayacucho \\
\hline PEVF09 & Ayacucho & 2.750 & Vicia faba & UNSCH-Ayacucho \\
\hline PEVF10 & Ayacucho & 2.750 & Vicia faba & UNSCH-Ayacucho \\
\hline PEVF11 & Ayacucho & 2.750 & Vicia faba & UNSCH-Ayacucho \\
\hline PEPSM12 & $\begin{array}{l}\text { Lima } \\
\text { (Cañete) }\end{array}$ & 39 & $\begin{array}{l}\text { Pisum sativum } \\
\text { macrocarpum }\end{array}$ & $\begin{array}{l}\text { LEMYB Marino } \\
\text { Tabusso (2) }\end{array}$ \\
\hline PEPSM13 & $\begin{array}{l}\text { Lima } \\
\text { (Cañete) }\end{array}$ & 39 & $\begin{array}{l}\text { Pisum sativum } \\
\text { macrocarpum }\end{array}$ & $\begin{array}{c}\text { LEMYB Marino } \\
\text { Tabusso }\end{array}$ \\
\hline PEPSM14 & $\begin{array}{l}\text { Lima } \\
\text { (Cañete) }\end{array}$ & 39 & $\begin{array}{l}\text { Pisum sativum } \\
\text { macrocarpum }\end{array}$ & $\begin{array}{c}\text { LEMYB Marino } \\
\text { Tabusso }\end{array}$ \\
\hline PEPSM15 & $\begin{array}{l}\text { Lima } \\
\text { (Cañete) }\end{array}$ & 39 & $\begin{array}{l}\text { Pisum sativum } \\
\text { macrocarpum }\end{array}$ & $\begin{array}{c}\text { LEMYB Marino } \\
\text { Tabusso }\end{array}$ \\
\hline PEPSM16 & $\begin{array}{c}\text { Lima } \\
\text { (Cañete) }\end{array}$ & 39 & $\begin{array}{l}\text { Pisum sativum } \\
\text { macrocarpum }\end{array}$ & $\begin{array}{c}\text { LEMYB Marino } \\
\text { Tabusso }\end{array}$ \\
\hline PEPSM17 & $\begin{array}{l}\text { Lima (Nuevo } \\
\text { Imperial) }\end{array}$ & 252 & $\begin{array}{l}\text { Pisum sativum } \\
\text { macrocarpum }\end{array}$ & $\begin{array}{c}\text { LEMYB Marino } \\
\text { Tabusso }\end{array}$ \\
\hline PEPSM18 & $\begin{array}{l}\text { Lima (Nuevo } \\
\text { Imperial) }\end{array}$ & 252 & $\begin{array}{l}\text { Pisum sativum } \\
\text { macrocarpum }\end{array}$ & $\begin{array}{c}\text { LEMYB Marino } \\
\text { Tabusso }\end{array}$ \\
\hline PEPSM19 & $\begin{array}{l}\text { Lima (Nuevo } \\
\text { Imperial) }\end{array}$ & 252 & $\begin{array}{l}\text { Pisum sativum } \\
\text { macrocarpum }\end{array}$ & $\begin{array}{c}\text { LEMYB Marino } \\
\text { Tabusso }\end{array}$ \\
\hline
\end{tabular}

${ }^{(1)}$ Laboratorio de Rizobiología, Universidad Nacional de San Cristóbal de Huamanga, Ayacucho - Perú.

(2) Laboratorio de Ecología Microbiana y Biotecnología Marino Tabusso. Dpto. de Biología, Universidad Nacional Agraria La Molina (Tesis doctoral Nery Santillana)

\section{Efecto de Rhizobium sobre la germinación de} semillas de tomate (Lycopersicon esculentum)

Se emplearon semillas de tomate de la variedad Río Grande, desinfectadas e inoculadas con suspensiones densas (concentraciones de $10^{8}$ ufc. $\mathrm{mL}^{-}$ $\left.{ }^{1}\right)$ de cada una de las 19 cepas de Rhizobium a evaluarse. Para el tratamiento control, las semillas fueron sumergidas en agua destilada esterilizada. Todas las semillas fueron colocadas en recipientes que contenían arena esterilizada y humedecida. Se consideró 2 repeticiones por tratamiento.

A los 10 días, se determinó el porcentaje de germinación. Los resultados fueron analizados mediante la tabla de análisis de variancia y la prueba de rangos múltiple de Duncan $(\mathrm{P}=0.05)$ para comparaciones entre promedios de tratamientos.

Efecto de Rhizobium sobre el crecimiento de plantas de tomate (Lycopersicon esculentum)

El experimento se realizó en condiciones de cámara de crecimiento, utilizando suelo procedente del campus de la Universidad Nacional Agraria La Molina, cuyo análisis se indican en la Tabla 2.

Tabla 2. Caracterización de suelo del Campus Universitario de la UNALM.

\begin{tabular}{|c|c|c|}
\hline Análisis & Resultados & Método \\
\hline $\mathrm{pH}$ & 7.3 & Suelo:Agua relación $1: 1$ \\
\hline $\begin{array}{l}\text { Materia } \\
\text { Orgánica \% }\end{array}$ & 1.5 & Walkley y Black \\
\hline Fósforo (ppm) & 49.5 & $\begin{array}{l}\text { Método del Olsen } \\
\text { modificado }\end{array}$ \\
\hline Potasio (ppm) & 242 & $\begin{array}{l}\text { Extracción con acetato } \\
\text { de amonio }\end{array}$ \\
\hline Clase Textural & Franco arenoso & Método del hidrómetro \\
\hline
\end{tabular}

El suelo fue colocado en vasos de $300 \mathrm{~g}$ de capacidad. Las semillas, desinfectadas previamente, fueron inoculadas con suspensiones densas de las cepas de Rhizobium a evaluarse (concentraciones celulares a nivel de $10^{8}$ ufc. $\mathrm{mL}^{-1}$ ). Las semillas control (no inoculadas) fueron tratadas con agua destilada esterilizada.

El diseño utilizado fue completamente al azar con 21 tratamientos (19 cepas, un control sin inocular y sin fertilización química y un control con solo fertilización química (60-60-60 Unidades de NPK) y cuatro repeticiones.

Al inicio de floración, se procedió a la evaluación de la altura de la planta, el peso de la materia seca de la parte aérea, el peso de la materia seca de la raíz, peso de la materia seca total de la planta y el índice de efectividad de la inoculación (IEI) expresado en porcentaje (Davies et al, 2005), calculado mediante la siguiente expresión,

$I E I=\left[\frac{\text { Trat Inoculación }- \text { Control Sin Inoculación }}{\text { Control Sin Inoculación }}\right] \times 100$

Se realizaron el análisis de variancia y la prueba de Rangos Múltiples de Duncan $(\mathrm{P}=0.05)$ para determinar las diferencias entre tratamientos.

Los promedios para las tres variables agronómicas (altura, materia seca de la parte aérea y materia seca de la raíz) se ordenaron de mayor a menor y el 
promedio de estos órdenes se utilizó para establecer el valor de orden global (ranking) de los tratamientos estudiados.

\section{Resultados}

Efecto de Rhizobium sobre la germinación de semillas de tomate (Lycopersicon esculentum)

Se observaron diferencias significativas entre cepas y el control (Tabla 3). Las semillas inoculadas con las cepas PEVF02, PEVF03, PEVF05, PEVF08, PEVF09, PEVF10, PEPSM12, PEPSM15 y PEPSM17 presentaron $100 \%$ de germinación, superando significativamente al control que presentó $80 \%$ de germinación. El resto de cepas no fueron estadísticamente diferentes frente al control. Los resultados obtenidos muestran el efecto positivo de la aplicación de las cepas de Rhizobium a las semillas de tomate.

Tabla 3. Porcentaje de germinación de semillas de Lycopersicon esculentum var. Río Grande inoculadas con cepas de Rhizobium.

\begin{tabular}{|c|c|c|c|}
\hline \multirow{2}{*}{$\begin{array}{l}\text { Cepas } \\
\text { PEVF01 }\end{array}$} & \multicolumn{2}{|c|}{$\begin{array}{l}\% \\
\text { Germinación Cepas } \\
\text { semillas }{ }^{1}\end{array}$} & \multirow{2}{*}{$\begin{array}{l}\begin{array}{l}\% \\
\text { Germinación } \\
\text { semillas }\end{array} \\
100 \mathrm{a}\end{array}$} \\
\hline & $90 \mathrm{ab}$ & PEPSM12 & \\
\hline PEVF02 & $100 \mathrm{a}$ & PEPSM13 & $90 \mathrm{ab}$ \\
\hline PEVF03 & $100 \mathrm{a}$ & PEPSM14 & $90 \mathrm{bc}$ \\
\hline PEVF04 & $60 \mathrm{~d}$ & PEPSM15 & $100 \mathrm{a}$ \\
\hline PEVF05 & $100 \mathrm{a}$ & PEPSM16 & $90 \mathrm{bc}$ \\
\hline PEVF06 & $80 \mathrm{bcd}$ & PEPSM17 & $100 \mathrm{a}$ \\
\hline PEVF07 & $80 \mathrm{bcd}$ & PEPSM18 & $70 \mathrm{~cd}$ \\
\hline PEVF08 & $100 \mathrm{a}$ & PEPSM19 & $70 \mathrm{bc}$ \\
\hline PEVF09 & $100 \mathrm{a}$ & & \\
\hline PEVF10 & $100 \mathrm{a}$ & & \\
\hline PEVF11 & $70 \mathrm{~cd}$ & & \\
\hline Control & $80 \mathrm{bcd}$ & & \\
\hline
\end{tabular}

${ }^{1}$ datos transformados a arcoseno de la proporción (porcentaje/100) de germinación antes de análisis. Tratamientos seguidos por la misma letra no difieren significativamente de acuerdo a la prueba de Rangos Múltiples de Duncan $(\mathrm{P}=0.05)$

Efecto de Rhizobium sobre el crecimiento de plantas de tomate (Lycopersicon esculentum)

La variable altura de planta no presentó diferencias significativas entre tratamientos (Tabla 4), mientras que la materia seca de la parte aérea y materia seca total si presentaron diferencias entre tratamientos. Se observó que el tratamiento con fertilización química y con las cepas PEVF01, PEVF02 y PEVF08 superaron significativamente al control sin inocular, el tratamiento con fertilización química incrementó la materia seca de la parte aérea en $88 \%$ y la materia seca total en $81 \%$ respecto al control. Las plantas tratadas con las cepas incrementaron la materia seca de la parte aérea entre 56 y $58 \%$ y la materia seca total entre 64 y $70 \%$ respecto al control (Figura 1).

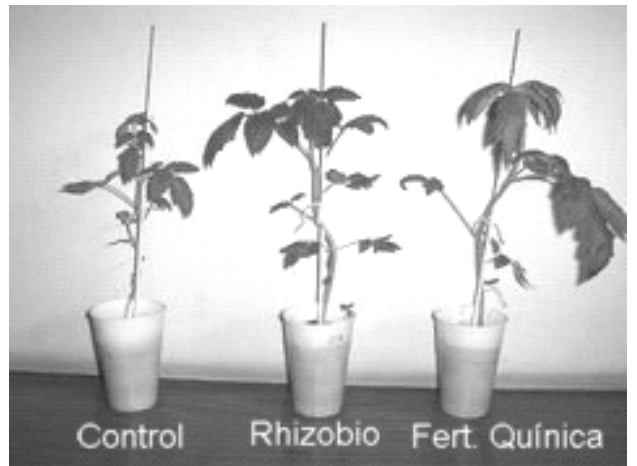

Figura 1. Efecto de rizobios en el crecimiento de Lycopersicon esculentum.

La materia seca de la raíz muestra diferencias significativas entre las plantas inoculadas con las cepas PEVF01 y PEVF08 frente al control sin inocular, con incrementos de $159 \%$, sin embargo, se observó que las cepas PEVF02, PEVF03, PEVF04, PEPSM12 y PEPSM19 presentaron incrementos entre 49 a $124 \%$ de la materia seca radicular frente al control sin inocular aunque sin diferencias significativas.

\section{Discusión}

En la actualidad, la agricultura sustentable, plantea mejorar la eficiencia de la fijación del nitrógeno mediante el uso de plantas leguminosas y rizobios competitivos, capaces de ser usados en biorremediación y fitorremediación y de esta manera extender las ventajas de la simbiosis a otros cultivos; en tal sentido, las investigaciones se han orientado al estudio del rizobios como promotor del crecimiento de plantas leguminosas y no leguminosas, proceso conocido como capacidad PGPR.

Previamente se demostró que algunas de las cepas de Rhizobium utilizadas en este estudio mejoraron el rendimiento del cultivo de haba ( $P$. sativum) a nivel de campo (Nuñez et al., 2005). En el presente trabajo, se verifica que ciertas cepas de Rhizobium, pueden estimular la germinación de semillas de tomate $\mathrm{y}$ promover su crecimiento.

El 47\% de las cepas de rizobios (cepas PEVF02, PEVF03, PEVF05, PEVF08, PEVF09, PEVF10, PEPSM12, PEPSM15 y PEPSM17) presentaron efecto estimulante sobre las semillas de tomate, resultando en una mejor germinación, posiblemente debido a la habilidad de los rizobios para producir hormonas como el ácido indol acético, ácido giberélico y citoquininas, sustancias reguladoras del crecimiento de las plantas (Dey et al., 2004; Yanni et al., 2001). Perrine et al. (2004) encontraron cepas de Rhizobium 
productoras de altas concentraciones de ácido indol acético que estimularon el crecimiento de semillas de arroz. Indican que su efecto es similar al ácido indol acético exógeno.

nitrógeno (Wang et al., 2002). Antoun et al. (1998), reportaron algunas evidencias directas de la colonización de raíces y la actividad PGPR de rhizobios con no leguminosas.

Se observó también, mayores incrementos de la materia Tabla 4. Altura, materia seca de parte aérea, materia seca de raíz, materia seca total e índice de efectividad de la inoculación de plantas de tomate (Lycopersicon esculentum) con cepas de Rhizobium.

\begin{tabular}{|c|c|c|c|c|c|c|c|c|c|}
\hline Cepas & $\begin{array}{l}\text { Altura } \\
(\mathrm{cm})\end{array}$ & $\begin{array}{l}\text { IEI } \\
\%\end{array}$ & $\begin{array}{l}\text { Materia seca } \\
\text { parte aérea }^{1} \\
\text { (mg/maceta) }\end{array}$ & $\begin{array}{l}\text { IEI } \\
\%\end{array}$ & $\begin{array}{c}\text { Materia seca } \\
\text { raíz }{ }^{1} \\
\text { (mg/maceta) }\end{array}$ & $\begin{array}{l}\text { IEI } \\
\%\end{array}$ & $\begin{array}{c}\text { Materia } \\
\text { seca } \\
\text { total }^{1} \\
(\mathrm{mg} / \text { macet } \\
\text { a })\end{array}$ & $\begin{array}{l}\text { IEI } \\
\%\end{array}$ & Orden \\
\hline PEVF01 & 41 & - & $492.5 \mathrm{ab}$ & 56 & $110.0 \mathrm{a}$ & 159 & $602.5 \mathrm{ab}$ & 69 & 5 \\
\hline PEVF02 & 42.7 & - & $492.5 \mathrm{ab}$ & 56 & $95.0 \mathrm{ab}$ & 124 & $587.5 \mathrm{a}-\mathrm{c}$ & 64 & 4 \\
\hline PEVF03 & 43.5 & - & $372.5 \mathrm{~b}-\mathrm{e}$ & 18 & $75.0 \mathrm{a}-\mathrm{c}$ & 76 & $447.5 \mathrm{~b}-\mathrm{e}$ & 25 & 6 \\
\hline PEVF04 & 43.7 & - & $427.5 \mathrm{bc}$ & 36 & $70.0 \mathrm{a}-\mathrm{c}$ & 65 & $497.5 \mathrm{a}-\mathrm{d}$ & 39 & 3 \\
\hline PEVF05 & 44.5 & - & $365.0 \mathrm{~b}-\mathrm{e}$ & 16 & $57.5 \mathrm{a}-\mathrm{c}$ & 35 & $422.5 \mathrm{c}-\mathrm{e}$ & 18 & 7 \\
\hline PEVF06 & 43.2 & - & $340.0 \mathrm{c}-\mathrm{e}$ & 8 & $60.0 \mathrm{a}-\mathrm{c}$ & 41 & $400.0 \mathrm{de}$ & 12 & 11 \\
\hline PEVF07 & 43.5 & - & $347.5 \mathrm{c}-\mathrm{e}$ & 10 & $60.0 \mathrm{a}-\mathrm{c}$ & 41 & $407.5 \mathrm{de}$ & 14 & 10 \\
\hline PEVF08 & 44.7 & - & $497.5 \mathrm{ab}$ & 58 & $110.0 \mathrm{a}$ & 159 & $607.5 \mathrm{ab}$ & 70 & 1 \\
\hline PEVF09 & 44.5 & - & $302.5 \mathrm{c}-\mathrm{e}$ & - & $57.5 \mathrm{a}-\mathrm{c}$ & 35 & $360.0 \mathrm{de}$ & 1 & 12.5 \\
\hline PEVF10 & 44.2 & - & $347.5 \mathrm{c}-\mathrm{e}$ & 10 & $57.5 \mathrm{a}-\mathrm{c}$ & 35 & $405.0 \mathrm{de}$ & 13 & 9 \\
\hline PEVF11 & 39.7 & - & $307.5 \mathrm{c}-\mathrm{e}$ & - & $55.0 \mathrm{bc}$ & 29 & $362.5 \mathrm{de}$ & 1 & 20 \\
\hline PEPSM12 & 42.2 & - & $272.5 \mathrm{de}$ & - & $63.30 \mathrm{a}-\mathrm{c}$ & 49 & $327.5 \mathrm{de}$ & - & 17.5 \\
\hline PEPSM13 & 43 & - & $322.5 \mathrm{c}-\mathrm{e}$ & 2 & $55.0 \mathrm{bc}$ & 29 & $377.5 \mathrm{de}$ & 6 & 15 \\
\hline PEPSM14 & 46 & 2 & $407.5 \mathrm{~b}-\mathrm{d}$ & 29 & $50.0 \mathrm{bc}$ & 18 & $457.5 \mathrm{~b}-\mathrm{e}$ & 28 & 8 \\
\hline PEPSM15 & 44.2 & - & $265.0 \mathrm{de}$ & - & $22.5 \mathrm{c}$ & - & $287.5 \mathrm{e}$ & - & 19 \\
\hline PEPSM16 & 40.2 & - & $257.5 \mathrm{e}$ & - & $50.0 \mathrm{bc}$ & 18 & $307.5 \mathrm{e}$ & - & 21 \\
\hline PEPSM17 & 46.5 & 3 & $307.5 \mathrm{c}-\mathrm{e}$ & - & $50.0 \mathrm{bc}$ & 18 & $357.5 \mathrm{de}$ & - & 12.5 \\
\hline PEPSM18 & 44.2 & - & $312.5 \mathrm{c}-\mathrm{e}$ & - & $37.5 \mathrm{c}$ & - & $350.0 \mathrm{de}$ & - & 17.5 \\
\hline PEPSM19 & 40.5 & - & $290.0 \mathrm{c}-\mathrm{e}$ & - & $70.0 \mathrm{a}-\mathrm{c}$ & 65 & $360.0 \mathrm{de}$ & - & 16 \\
\hline Control & 45.2 & - & $315.0 \mathrm{c}-\mathrm{e}$ & - & $42.5 \mathrm{bc}$ & - & 357.5 de & - & 14 \\
\hline Fert.Quím. & 47.5 & 5 & $592.5 \mathrm{a}$ & 88 & $55.0 \mathrm{bc}$ & 29 & $647.5 \mathrm{a}$ & 81 & 2 \\
\hline
\end{tabular}
seca de la raíz con relación a la materia seca de la parte aérea, incrementos que superaron a la fertilización química. Dichos resultados concuerdan con autores como Mayak et al. (2004) quienes hacen mención sobre la habilidad de las cepas de rizobio para producir ACC diaminasa, compuesto que reduce el nivel de etileno en las raíces de las plantas, incrementándose de esta manera la longitud y el crecimiento de las raíces. Mientras que Chabot et al. (1996), Yanni et al. (2001) y Perrine, et al. (2004) entre otros, sostienen que las moléculas promotoras del crecimiento como el ácido indol acético, las giberelinas y las citoquininas producidas por los rizobios, presentes ya sea en la rizósfera o en los tejidos de las plantas estimulan el mayor desarrollo de la raíz y realizan la capacidad de absorción de nutrientes de la raíz en beneficio de la planta no leguminosa. Gutiérrez \& Martínez (2001) encontraron incrementos de $42 \%$ de la materia seca de la parte aérea

El $37 \%$ de las cepas de Rhizobium evaluadas (cepas PEVF01, PEVF02, PEVF03, PEVF04, PEVF05, PEVF08 y PEPSM14) estimularon el crecimiento de las plantas de tomate. Al respecto numerosos estudios han demostrado el uso del rizobio como bacterias fijadoras del $\mathrm{N}_{2}$ y como promotoras del crecimiento de plantas no leguminosas. Por ejemplo $R$. leguminosarum bv trifolii y cepas de Bradyrhizobium se han encontrado en raíces de arroz y $R$. etli en raíces de maíz. Los rizobios pueden también introducirse y colonizar otras plantas, tal como sucede con Azorhizobium caulinodans en las raíces de la oleaginosa Brassica napus. Estas asociaciones entre rizobios y plantas no leguminosas pueden mejorar el crecimiento de las plantas aunque no se ha demostrado que sea mediante la fijación de y de $49 \%$ de la materia seca de raíz en plantas de maíz inoculadas con $R$. etli. Dichos autores mencionan que el efecto benéfico de $R$. etli en plantas de maíz puede deberse a los mecanismos descritos anteriormente.

Las cepas PEVF02 y PEVF08 tuvieron un efecto positivo significafvo en la germinación y en el crecimiento de las plantas de tomate, por lo cual podrían recomendarse como potenciales PGPR en este cultivo como alternativa para reducir el uso de fertilizantes químicos.

\section{Conclusiones}

1. Nueve cepas de rizobios (PEVF02, PEVF03, PEVF05, PEVF08, PEVF09, PEVF10, PEPSM12, PEPSM15 y PEPSM17) estimularon la 
germinación de las semillas de Lycopersicon esculentum.

2. Siete cepas de rizobios (PEVF01, PEVF02, PEVF03, PEVF04, PEVF05, PEVF08 y PEPSM14) promovieron el crecimiento de plantas de tomate (Lycopersicon esculentum).

3. Las cepas PEVF02 y PEVF08 estimularon significativamente la germinación de semillas y promovieron el crecimiento de plantas de Lycopersicon esculentum.

\section{Literatura citada}

Antoun H. Beauchamp Ch. \& Goussard N. 1998. Potential of Rhizobium and Bradyrhizobium species as plant growth promoting rhizobacteria on non legumes. Effect on radishes (Raphanus sativus L.). Plant and Soil. 204: 57-67

Carson K., Meyer J.M. \& Dilworth M. 2000. Hydroxamate siderophores of root nodule bacteria. Soil Biology \& Biochemistry. 32: 11-21.

Chabot R., Antoun H., Kloepper J.W. \& Beauchamp C.J. 1996. Root colonization of maize and lettuce by bioluminescent Rhizobium leguminosarum biovar. phaseoli. Appl. Environ. Microbiol. 62: 2767-2772.

Chakravarty U. \& Purkayastha R.P. 1984. Role of Rhizobitoxine in protecting soybean roots from Macrophomina phaseolina infection. Can. J. Microbiol. 30: 285-289.

Davies F.T., Calderón C.M. \& Huamán Z. 2005. Influence of Arbuscular Mycorrhizae Indigenous to Peru and a Flavonoid on Growth, Yield and Leaf Elemental Concentration of "Yungay" Potatoes. Hort Science. 40(2): 381-385

Dey R., Pal K., Bhatt D.M. \& Chauhan S.M. 2004. Growth promotion and yield enhancement of peanut (Arachis hypogaea L.) by application of plant growth-promoting rhizobacteria. Microbiological Research. 159: 371-394.

Essalmani H. \& Lahlou H. 2003. Mécanismes de bioprotection des plantes de lentile par Rhizobium leguminosarum contre Fusarium oxysporum sp. Lentis. C.R. Biologies. 326 : 1163-1173.

Gutierrez A. \& Martinez E. 2001. Natural endophytic association between Rhizobium ettli and maize (Zea mays L.). Journal of Biotechnology. 91: 117-126.
Hassan Dar G., Zargar M.Y. \& Beigh G.M. 1997. Biocontrol of Fusarium Root Rot in the Common Bean (Phaseolus vulgaris L.) by using Symbiotic Glomus mosseae and Rhizobium leguminosarum. Microb. Ecol. 34: $74-80$

Mayak S., Tirosh T. \& Glick B. 2004. Plant growthpromoting bacteria confer resistance in tomato plants to salt stress. Plant Physiology and Biochemistry. 42: 565572.

Mhadhbi H., Jebara M., Limam F. \& Elarbi Aouani M. 2004. Rhizobial strain involvement in plant growth, nodule protein composition and antioxidant enzyme activities of chickpea-rhizobia symbioses: modulation by salt stress. Plant Physiology and Biochemistry. 42: 717-722.

Nuñez, M., Santillana, N. \& Zúñiga Dávila, D. 2005. Evaluación de cuatro cepas de Rhizobium en Vicia faba L. var. Rojo Mantaro en condiciones de campo. Naturaleza y Desarrollo. 3(2): En prensa.

Perrine F., Rolfe B., Hynes M. \& Hocart C. 2004. Gas chromatography-mass spectrometry analysis of indolacetic acid and tryptophan following aquerous chloroformate derivatisation of Rhizobium exudates. Plant Physiology and Biochemistry. 42: 723-729.

Rodriguez H. \& Fraga R. 1999. Phosphate solubilizing bacteria and their role in plant growth promotion. Biotechnology Advances. 17: 319-339.

Sessitsch J., Howieson X., Perret H., Antoun H. \& MartinezRomero E. 2002. Advances in Rhizobium Research. Critical Reviews in Plant Sciences. 21, Issue 4, 1 : 323378.

van Rossum D., Muyotcha A., van Verseveld H.W., Stouthamer A.H. \& Boogerd F. 1994. Siderophore production by Bradyrhizobium spp. strains nodulating grundnut. Plant and Soil. 163: 177-187.

Wang T., Martinez J. \& López I. 2002. Rhizobium y su simbiosis con plantas. Monografía. Centro de Investigación sobre Fijación de Nitrógeno. Universidad Nacional Autónoma de México.

Yanni Y., Rizk R., Fattah F.K. \& Squartine A. 2001. The beneficial plant growth-promoting association of Rhizobium leguminosarum bv. trifolii with rice root. Australian Journal of Plant Physiology. 28: 845-870.

\footnotetext{
${ }^{1}$ Laboratorio de Rhizobiología. Universidad Nacional de San Cristóbal de Huamanga, Ayacucho. nerysantillana@yahoo.es

${ }^{2}$ Dpto de Biología. Universidad Nacional Agraria La Molina, Lima. carellano@lamolina.edu.pe

${ }^{3}$ Laboratorio de Ecología Microbiana y Biotecnología. Universidad Nacional Agraria La Molina, Lima. dzuniga@lamolina.edu.pe
} 